\title{
La educación británica en el campo de la bibliotecología y ciencias de la información
}

\section{Jesús Lau \\ RESUMEN}

Elobje ti vo del ar tícu lo es des cribir elsistema educa tivo de bibliote colo gíaycienciasdela información (BCI) del Rei no Uni do. Al momen to de re dac tar lo se tuvo en cuen ta las ne ce si da des de las per so nas que pu die ran estar in te re sa das en es tu diar en este país, y se cun dariamente en las involucradas en la formación de profesionales de este campo. El artículo ofrece una introducción a la evolución edu ca ti va de BCI, de fine los di fe ren tes gra dos ofre ci dos, proce di mien to de se lec ción de es tu dian tes, ad mi sión y fi nan cia mien to, y los con tro les de ca li dad aca dé mi ca; más al gu nos co men ta rios so bre la adap ta ción que es tán efec tuan do las es cue las para en fren tar los cambios del mer ca do in for ma ti vo; y con clu ye dan do al gu nas de las ven tajas de la ins trucción bri tá ni ca. Alfi nal se ane xa un di rec to rio de las escuelas de BCI, incluyendo fecha de fundación, grados ofrecidos, número de maestros, y alumnos por especialidades.

\section{ABSTRACT}

The object of this ar tic le is to des cribe the li brary edu ca tio nal sys tem and the in for ma tion scien ces (BCI) of the Uni ted King dom. At the mo ment of wri ting this, it has ta ken into account the ne ces si ties of tho se per sons who were in te res ted in stud ying this coun try and se conda rily in tho se in vol ved in the for ma tion of pro fes sio nals in this field. The ar tic le offers an in tro duc tion to the edu ca tio nal evo lu tion of BCI, de fi nes the diffe rents gra des offe red, pro ce eds with the se lec tion of stu dents, ad mis sion and fi nan cing and the con trols of aca demic quality. Some of the commentaries about adaptation that are effecting the schools in confronting the changes of the information mar ket. It con clu des in gi ving some of the ad van ta ges of British ins truc tion. At the endis anne xed a di rec tory of the schools of BCI including date of foundation, grades offered, number of instructors and students by speciality.

\section{INTRODUCCION}

El Reino Unido, junto con el resto de las naciones angloparlan tes, es un país li der en el cam po de los ser vi cios in for ma tivos. Su in fluen cia es no to ria en los paí ses delCommonwalth, naciones que en el pasado fueron sus colonias. Pero a pesar de este papel internacional preponderante, en México se conoce poco so bre la edu ca ción bri tá ni ca, o cuan do me nos ésta fue la experiencia del suscrito antes de venir a este país.

Después de visitar algunas escuelas y de revisar folletos y programas de varias de ellas, se decidió redactar el presente ar tícu lo para lle nar par te de este va cío, ofre cien do un pa no rama sobre las características del sistema educativo británico en el área de la Bibliotecología y Ciencias de la Información (BCI). En ciertos párrafos se encontrarán comparaciones breves con el sistema norteamericano, sólo para ilustrar las características del primero. Cabe resaltar que la estructura educativa de Gran Bretaña es compleja para resumirse en unas cuantas páginas, ya que cada entidad geográfica es autónoma, las escuelas son flexibles en sus políticas y la instruc ción es im par ti da por dos ti pos de ins ti tu cio nes: uni ver sida des y poli téc nicos; au na do a la par ti ci pa ción re gu la do ra de institucionesgubernamentales y de asociaciones profesionales. Por lo tanto el lec tor de be rá te ner pre sen te que el nú me ro de similitudes entre los grados y planes académicos, puede en algunos casos ser igual a las diferencias.

\section{ORIGENES DE LA PROFESION.}

Para ini ciar, es con ve nien te de cir que el Rei no Uni do está divididopolíticamente en tres reinos: Inglaterra, Es co cia y Gales, más Irlanda del Norte. Cada región tiene sus propios sistemas de servicios informativos, incluyendo su propia bibliotecanacional, as pectos en los que no se en tra ráen de talle; sólo diremos que Inglaterra tiene trece escuelas de BCI, Escocia dos, Irlanda del Norte una y Gales otra (véase apéndice de lista de es cue las). La po bla ción de todo el país es casi de 60 millones, y viven en un área geográfica que equivale a un octavo del territorio mexicano.

La educación en el campo de la BCI se remonta a finales del siglo pa sa do, cuan do la ca pa ci ta ción del per so nal de bi blio tecas se lograba mediante cursos nocturnos, de verano, o por correspondencia y con entrenamiento en el servicio. La educa ción for mal, como opues ta a la edu ca ción fue ra del aula, se ini ció has ta 1932, fe cha en que el Uni ver sity Colle ge Lon don ofre cióla es pe cia li dad de bi blio te co lo gía a ni vel téc ni co. Cator ce años más tar de al gu nos co le gios de co mer cio y tec no logía establecieron también programas técnicos (Saunders).

En Estados Unidos de Norteamérica, por otro lado, la enseñanzabibliotecológica se con so li dó en una ca rre ra for mal alrededor de 1920. La razón de este lento proceso evolutivo británico hacia la instrucción escolarizada se debe a cuestiones históricas que se re mon tan a los al bo res de la Re volu ción Industrial. 
Desde finales del siglo XVIII, la mayoría de las profesiones británicas se aprendían mediante capacitación en el trabajo, así se educaban los abogados, arquitectos e ingenieros, entre otros. Los aprendices de todas estas disciplinas pasaban un lar go pe río do de en tre na mien to en los cen tros de tra bajo. Las universidades de la época, Oxford y Cambridge, consideraban que sus objetivos eran únicamente cultivar seres humanos y no enseñar ofi cios para ga nar se la vida, de jan do la educación profesional en manos de las asociaciones de cada especialidad.

Ante esta necesidad nació la Library Association (LA) en 1877 , y muy pron to, en 1880 , dio los pri me ros pa sos para entrenar a sus miembros, creando un sistema de exámenes secuenciales para obtener el certificado de competencia en la rama, e imponiendo una serie de requisitos para ser candidato a tal reconocimiento. Así, a partir de esa época, formó al personal de los centros bibliotecarios, función que limitó en 1962, es decir, ochenta años más tarde; para después renunciar a ella casi por completo a principios de los años setenta, cuando se consideró que los bibliotecarios debían ser educados por las universidades (Thompson). Esta renuncia a la educación de los socios no fue aceptada únicamente por la LA, sino también por asociaciones de otras especialidades como las del magisterio, contaduría pública, y enfermería.

Hasta 1964 existían diez escuelas de bibliotecología, y de ellas sólo la de University College London ofrecía estudios de tiempo completo para el diploma, cuyos egresados estaban exentos de la mayoría de las evaluaciones de la LA. Las demás instituciones se dedicaban a preparar estudiantes de me dio tiem po para to mar exá me nes de la aso cia ción, en programas que duraban de uno a dos años.

Gran parte de las escuelas, como ya se mencionó, estaban principalmente en colegios técnicos, ahora convertidos en politécnicos. Para mediados de los años sesenta la demanda debibliotecarios se incrementó notablemente, así los centros educativos crecieron de unos tres maestros a aproximadamen te quin ce; y los alum nos au men ta ron en pro me dio de 150 a 200, pero aho ra de tiem po com ple to; dos es cue las te nían inclu si ve más de 300 es tu dian tes. Para man te ner la ca li dad académica en esta etapa de crecimiento, la LA y el Council for National Academic Awards (CNAA) exigieron como mínimo un maes tro de tiem po com ple to por cada diez es tu dian tes (Bramley).

Como resultado de la expansióneducativa y la autonomíade las escuelas, se crearon programas para una variedad de grados y especialidades. La situación presente es que varios de los politécnicos y las universidades tienen una variedad de alumnos, que comprende desde los que estudian licenciatura (bachelor), di plo ma o maes tría, has ta los que in ves ti gan a nivel doctoral o postdoctoral. Las escuelas forman anualmente un promedio de mil personas en sus diferentes grados, las cuales substituyen paulatinamente a gran parte del personal que co men zó a tra ba jar en las dos dé ca das an te rio res sin preparación tan formal en el área. Así, poco a poco, la fuerza de trabajo se transforma en un grupo con un ni vel míni mo de licenciatura. El personal profesional en sus diversos grados, que aho ra se en cuen tra tra ba jan do, as cien de a $22 \mathrm{mil}$ (Mo ore y Kempson).

\section{LOS DIFERENTES GRADOS}

Los acelerados cambios tecnológicos, en los campos de la computación, electrónica y las telecomunicaciones, han hecho que las es cue las re duz can su re sis ten cia al cam bio. El enfoque desproporcionadohacia las técnicas, en lugar de hacia los principios, empieza a desaparecer en las instituciones aunque, no obstante, existen todavía centros educativos que conservan el enfoque tradicional.

Los pro gra mas, así como los nom bres de va rias es cue las empezaron a cambiar a finales de la década pasada. Algunas de éstas ofrecen simultáneamente cursos separados para bibliotecología e información. En otras como se notará en el apéndice, ofrecen programas exclusivamente para estudios de la información, poniendo énfasis en programación, recuperación automatizada de información, telemática y materias íntimamenterelacionadas contecnologíainformativa. Algunas escuelas, inclusive, empiezan a competirconlases pecialidades de computación y administración, que a futuro planean fusiones de programas conjuntos entre tales áreas.

A pesar de estos cambios, las escuelas británicas están sufriendo los efectos de las nuevas tecnologías, aunados a los de la recesión económica. En consecuencia los británicos quie ren evitar la ex perien cia nor te ame ri ca na de ce rrarescuelas sin nin gu na pla nea ción (LA), por lo que el go bier no rea lizó un estudio para fusionar escuelas de BCI, acción tomada ya para las de arquitectura, y a las universidades mismas se les anunció que existe tal posibilidad. El estudio recomendó también que las escuelas reorientaran los programas académicos no sólo a las necesidades de las bibliotecas, sino también a las de la industria informativa, quen contrata a la mayor parte de los egresados orientados a tecnología informativa. Entre otras recomendaciones que vale la pena mencionar, se sugirió que Loughborough Technical College ce rra ra su pro gra ma, y que la Es cue la de Li ver pool po dría seguir un curso similar. Asimismo, fue recomendada la fusión de las tres escuelas de Londres en una. Otro aspecto incluido en el reporte es el cuestionamiento de cambios organizacionales y académicos que se han realizado en Strathclyde y en el Politécnico del Norte de Londres.

Los gra dos ofre ci dos por las es cue las, has ta la fe cha, pue den clasificarse en seis ti pos, los que a su vez se am plían en un gran número de especialidades. El cuadro adjunto resume dichos grados, describiendo su duración mínima. En los siguientes párrafos se encontrará mayor información respecto a ellos y en el apéndice se listan casi todas las especialidades. 


\begin{tabular}{lll}
\hline \multicolumn{2}{|c}{ Clasificación de grados } \\
\hline Grado & Abrev. & Duración \\
\hline Nivel técnico & Dip & $\begin{array}{l}\text { Dos años (tiende a } \\
\text { desaparecer) }\end{array}$ \\
Licenciatura & BA,BSc & Tres o cuatro años \\
Diploma de postgrado & PDip & Un año académico \\
Maestría & MA, MSc & Un año calendario \\
Maestría en filosofía & MPhil & $\begin{array}{l}\text { Un año de inscrip. y } \\
\text { cuatro para tesis }\end{array}$ \\
Doctorado & PhD & $\begin{array}{l}\text { Dos a tres de inscrip. } \\
\text { y 5 años para tesis }\end{array}$ \\
& & Mínimo un año
\end{tabular}

\section{Nivel técnico y capacitación}

Como se mencionó antes, este nivel fue el primero en ofrecerse, pero ahora sólo lo tiene la escuela de Loughborough Technical Co lle ge. Su du ra ción es de dos años y en él se forma personal de apoyo administrativo. El programa como ya señalamos, está a punto de desaparecer, quizá por la capacitación bibliotecaria que realizan el Business Educational Council y los llamados City Guilds, que son cen tros de ca pacitación para adul tos o jó ve nes. Casi to das las gran des ciu dades tie nen es tos pro gra mas de en se ñan za de ofi cios, fi nan ciados por los gobiernos de los condados.

\section{Licenciatura BA, BSc}

La licenciatura en bibliotecología BA incluye principalmente materias humanísticas, bibliotecología, y en menor escala ciencias de la información. La de ciencias de la información BSc, por otro lado, hace énfasis en automatización, materias relacionadas con ciencias puras y aplicadas, y en menor grado bibliotecología. Ambas carreras pueden durar de tres a cuatro años, siendo el de tres el más común. La variación radica en el tiempo dedicado a la práctica, por ejemplo, si se prevee un año para esto, la duración será de cuatro.

Algunas escuelas ofrecen carreras con dos especialidades, donde la se gun da es un tema dis tin to a BCI, ésta pue de ser en ciencias, humanidades, arte o ciencias sociales, finalmente; los egresados de nivel licenciatura equivalen anualmente a un $40 \%$ del total de nuevos profesionales, a diferencia de EUA, donde la mayor parte de los profesionales se forman a nivel postgrado.

\section{El PDip}

Es un curso que dura un año académico, o sea ocho o nueve meses, y es ofrecido principalmente por los politécnicos. Se le considera inferior a la maestría, aunque en algunos casos

puede ser de la misma calidad. El programa generalmente es orientado a biblioteconomía, pero existen algunos para tecnologíainformativa.Otracaracterística es que al terminarlos cursos los estudiantes presentan un reporte, y no una tesina como los alumnos de maestría.

\section{Maestrías}

Estas se dividen principalmente en MA en artes; MSc en ciencias sociales; MSc en ciencia; y ME (Mature Entry) para cualquier área de elec ción por que es para es tu dian tes con experiencia; y finalmente el MLS que tam bién es en ar tes. Casi todos estos programas duran un año calendario, a diferencia del diploma que se realiza en ocho o nueve meses. El MA está diseñado para alumnos con formación humanística, quie nes son lla ma dos bi blio te ca rios en toda la ex ten sión de la palabra. Sus cursos in clu yen, como el BA, ma te rias bi blio tecológicas principalmente.Los dos MSc hacen én fa sis en tecnología informativa. El MLS, sin embargo, es una maestría con orientación intermedia entre bibliotecología e información, y comprende un mayor número de materias, que se toman du ran te un año y me dio. La maes tría de no mi na da ME es unaalternativa para per so nas con es tu dios pre vios, y con experiencia mínima de dos años en el campo. Los estudiantes pue den se lec cio nar las ma te rias de acuer do con sus in te re ses, aunque deben tener un balance de ellas, según sea la orientación escogida (Wilson).

Los participantes en cualquiera de los puntos descritos, deben pre sen tar una te si na que pre pa ran de tiem po com ple to en los tres últimos meses del año académico. La titulación de alumnos es masiva, ya que casi el cien por cien de los alumnos termina la disertación.

Las maestrías también se pueden obtener mediante investiga ción, es de cir, sin to mar cur so al gu no. Quie nes so li ci tan tal modalidad, que son muy pocos, deben ser personas con buena for ma ción y ex pe rien cia pre via en el área. El tra ba jo de investigación es a nivel de tesis, y no de tesina, la cual hay que defender en examen recepcional. Esta opción es apropiada para quienes trabajan, ya que sólo visitan la institución educativa con irregularidad.

\section{Maestría en Filosofía MPhil}

Este es un gra do in ter me dio en tre las otras maes trías y el doctorado. La diferencia del MPhil con este último es que la investigación puede ser descriptiva y no necesariamente una nueva apor ta ción para la ex pan sión del co no ci mien to. La investigación, sin embargo, debe seguir la mayoría de los lineamientos del nivel doctoral. La inscripción en la universidad o politécnico es obligatoria durante un año y normalmente se dan cuatro para presentar la tesis y el examen profesional.

\section{Doctorado PhD}

Los doctorados usualmente se realizan sobre te mas que caen den tro de más de una dis ci pli na, por el ca rác ter mul ti dis ci plina rio de la in for ma ción y la bi blio te co lo gía. La li mi ta ción temática en algunasfaculta des depende de la dis ponibilidad de 
supervisores y de los equipos disponibles. Los programas doctorales no incluyen ningún curso, o introducción previa para iniciar la investigación. Al comenzar, el alumno debe prepararse autodidácticamente en metodología de la investigación, en el tema de trabajo, y en algunos casos debe familiarizarse con las nuevas tecnologías, como la computadora. Esta fase es di fí cil, más no im po si ble, para alum nos de paí ses en vías de desarrollo (o novicios en general), porque pocos es tu dian tes han in ves tiga do o publica do an tes, y porque a veces eligen un tema del que no tienen antecedentes.

El tiempo dedicado a la primera fase, repitiendo, depende de la experiencia del candidato, aunque normalmente casi todo el primer año se dedica al es tu dio de la me to do lo gía y si multánea men te se de li mi ta el tó pi co de in ves ti ga ción. Los doc torados en EUA incluyen a cambio cursos tipo seminario para el primer cicloacadémico del programa, lo que facilita la intro duc ción al tra ba jo doc to ral pero, a su vez, es una des ven ta ja para los pocos que tengan experiencia en investigación.

Otra dife ren cia, en tre lascasas de es tu dios nor te ame ricanasy bri tá ni cas, es que es tas úl ti mas ofre cen úni ca men te el doc torado por investigación, denominado Philosophical Doctorate $(\mathrm{PhD})$, y las instituciones norteamericanas ofrecen dos tipos de doctorados, el PhD y el llamado Doctorate (Dr). El segundo se obtiene con un programa de estudios flexible, cuya investigación puede ser de tipo descriptivo, con menor én fa sis en la for ma ción in ves ti ga do ra, mien tras que el doc tora do en fi lo so fía se hace sólo me dian te in ves ti ga ción cien tífica.

En cuanto a la duración, en Gran Bretaña debe pagarse un mínimo de dos a tres años de colegiatura, o más, si se continúan usando las instalaciones universitarias, y se tiene cinco años para presentar la tesis. Estos períodos casi se duplican cuando el programa se realiza de medio tiempo. A este respecto conviene mencionar que, en algunos casos, las universidades permiten realizar parte de los estudios fuera de ésta después del primer año de investigación. La concesión depende del tema de estudio, y de las instalaciones o equipos disponibles en el lugar foráneo propuesto.

\section{Postdoctorado}

Las es cue las uni ver si ta rias son las úni cas que ofre cen este nivel. Los candidatos obviamente son doctores que realizan una investigación con la supervisión de un miembro del personal aca dé mi co, por un pe río do de uno o más años. El cri terio para efectuar este grado varía de acuerdo con la institución. Quienes lo realizan son personas directamente involucradas en la investigación o en la docencia, que toman una estancia en otra universidad, para adquirir mayor experiencia, por lo que el número de participantes es reducido.

\section{ELECCION DE LA ESCUELA}

En el Reino Unido, al igual que en otros países, hay escuelas con me jor ni vel que otras, de sa for tu na da men te no exis ten estudios comparativos publicados al respecto, por lo que no es posible listarlas en orden de calidad académica. La persona interesada tiene que realizar su propia decisión con los datos disponibles, los cuales no siempre serán suficientes u objetivos. Los factores que, tal vez, se deben tomar más en cuenta son:

1. El prestigio del personal académico, para lo cual la cantidad y calidad de los trabajos publicados, así como la participación en eventos internacionales, pueden servir de indicadores.

2. La disponibilidad de estudios de doctorado, porque esto indicará que el profesorado tiene al tos es tu dios y que la ins titución cuenta con buenos recursos.

3. Los programas universitarios de postgrado tienden a ser mejores que los politécnicos, porque los primeros poseen mayores recursos para esta especialidad.

4. La ubicación geográfica es importante si se desea aprovechar as pec tos cul tu ra les en este país. Las ciu da des de ta ma ño mediano ofrecen mejores ventajas.

Para elegir la institución también es recomendable consultar el directorio internacional de escuelas de este campo, publicado recientemente por IFLA (Fang), el cual da información sobre el número de maestros, alumnos, trabajos publicados y el tipo de instalaciones, como biblioteca y sistemas de cómputo disponibles. Con un breve análisis comparativo de las escuelas se puede determinar cuáles son las más apropiadas de acuerdo al área de interés que se tenga en mente.

\section{ADMISION}

Lasins ti tu ciones edu cativas re ciben con gus to a los es tu diantes extranjeros, ya que sus colegiaturas son una fuente extra de in gre sos, en mo men tos en que re sien ten la cri sis eco nó mica in ter na cio nal. A pe sar del in te rés eco nó mi co que és tas pudie ran te ner, la edu ca ción bri tá ni ca es poco co mer cial, a di ferencia de algunas universidades norteamericanas. El candidato a estudiante empieza a notarlo cuando solicita información para inscribirse, porque la folletería tiende a cubrir lo básico, y son pocas las instituciones que cuentan con documentos publicitarios. La explicación de esto es que la edu cación su periorbritánicaes es tatal, ex cep to una uni versidad que es privada.

Las es cue las de bi blio te co no mía eli gen a sus es tu dian tes mediante un procedimiento largo, pero en esta sección sólo haremos referencia al procedimiento de admisión para programas de postgrado. Los candidatos para estudiar maestría, deben tener la pre sen ta ción de una so li citud de in gre sos doce meses an tes del pe río do lec ti vo a es tu diar. El de par ta men to o escuela preselecciona a los can di da tos con base en sus ca li ficaciones. Posteriormente, los preseleccionados requieren tener una en tre vis ta, en la cual se les cues tio na so bre sus in te reses, y de acuerdo con los resultados son aceptados o rechazados. Los candidatos extranjeros están exentos de la en tre vis ta, y los lími tes de tiem po son fle xi bles, e igual men te acep tan los es tu dios de li cen cia tu ra como equi va len tes al $b a$ chelor británico. 
Los aspirantes a doctorado deben presentar previamente un es bo zo del pro ble ma que les in te re sa es tu diar, ade más del $c u$ rrículum vitae, que es importante porque las instituciones lo uti li zan para de ter mi nar la ca pa ci dad del es tu dian te. Otro criterio que se aplica para la aceptación es la disponibilidad, en eldepartamento o escue la, de un su per vi sor con co no ci mientos del problema propuesto. Normalmente los estudiantes ex tran je ros, y la ma yo ría de los bri tá ni cos, son acep ta dos inicialmente para maestría de investigación MPhil. Después de unos meses el estudiante debe presentar el anteproyecto de tesis, con el cual se decide si se le otorga la transferencia al doctorado. Otro requisito indispensable es obtener un buen puntaje en el examen de inglés que aplica el Consejo Británico.

\section{FINANCIAMIENTO}

Como se mencionó en el párrafo anterior, la educación es casi completamente estatal. Aparte del pago de colegiaturas, los estudiantes británicos reciben becas de manutención. Hasta 1981 las cuotas de inscripción eran iguales para extranjeros y nacionales, pero actualmente los primeros deben pa gar el do ble; aun así, és tas pa re cen ser más ba jas que en los Estados Unidos. El cos to de la ins crip ción anual es al re de dor de 3,500 libras. Esta cantidad puede ser disminuida si se obtiene una beca que otorga el gobierno británico a personas extranjeras, que pretendan obtener un grado a base de investi ga ción. La beca con sis te en una dis mi nu ción de las co le gia turas, hasta la cantidad establecida para los británicos; este descuento equivale a un cincuenta por ciento en el área de BCI. Los requisitos para obtenerla son: que el alumno tenga muy buena capacidad académica, y que haya estudiado al me nos un año an tes en Gran Bre ta ña, ade más de que no ten ga beca de otro organismo británico, sin importar si recibe apoyo económico de otro país. En 1985 se otorgaron unas ochocientas becas de este tipo, que son de beneficio también para Gran Bretaña, pues de esta manera amplía la investigación nacional. El Consejo Británico, como es ampliamente conocido, también ofrece becas anualmente para este campo, que cubren gastos de inscripción y manutención.

\section{CONTROL ACADEMICO}

La calidad académica inglesa incluye varios controles. Primero, las es cue las de ben ser re co no ci das por el go bier no; segun do, los pro gra mas de ben ser apro ba dos por la LA y las escuelas de los politécnicos requieren, además, la aprobación de la CNAA. Esta va li da ción de pro gra mas es si mi lar a la que brinda la American Library Association, con la diferenciade que en el Reino Unido, las escuelas no pueden existir legalmente si no tienen aprobados sus planes académicos.

El con trol aca dé mi co se re fle ja aun en la eta pa fi nal de la formación de los futuros profesionales. Después de que los alumnos de licenciatura o maestría son examinados por los maestros de la propia escuela, sus trabajos y exámenes son enviados a un examinador externo de otra escuela, quien tiene la última palabra para aprobar o reprobar. En cuanto a los estudiantes de maestría se envía, además, la tesina al exami- nador, quien puede pedir correcciones al trabajo e inclusive hacer un examen oral si lo considera pertinente.

Para los doc to ra dos o maes trías por in ves ti ga ción, el proce so varía un poco por las características de este tipo de estudios. La ela bo ra ción de la te sis se rea li za bajo la su per vi sión de un maestro de la institución con especialidad en el tópico de la investigación, quien termina su función cuando el alumno con clu ye el tra ba jo, ya que no toma par te en el exa men re cepcional. Para esta etapa final, se nombra a otro maestro de la misma es cue la como si no dal in ter no, y se in vi ta a una per sona ajena a la escuela como examinador externo, quien funge como presidente del jurado. Ambos dan el veredicto final de la calidad de la investigación con el examen recepcional. Este procedimiento permite, de hecho, que el supervisor sea también calificado, según sea el resultado.

El examinador externo es usualmente un experto en la materia, debido a esto, los grados por investigación se miden extraoficialmente de acuerdo con la calidad y el renombre del sinodal externo, quien no debe tener relación previa con el sustentante, y si es así, éste es cambiado por otra persona.

\section{CONCLUSIONES}

Las es cue las británicas presen tan ven tajas y des ven tajas. Entre las segundas se encuentran que es tán en un país geo grá ficamente pequeño, comparado con los Estados Unidos. Por otro lado, Gran Bretaña ya no es el imperio que dominabaun tercio del globo terráqueo; después de la Segunda Guerra Mundial las colonias ganaron su independencia, y con ello dejaron de contribuir al erario británico. Esto repercute, a su vez, en que mu chos pro gra mas de in ves ti ga ción sean orien tados al prag ma tis mo. Por otro lado, es tas des ven ta jas, son a su vez factores que han influido para que los británicos sean ahorrativos. La comunidad académica tiene un alto sentido de economía, lo cual es un contraste con la sociedad de consumo de Norteamérica. La optimización hace que los recursos limitados, que no lo son si los comparamos con los de México, sean suficientes para mantener un liderazgo en investigación y desarrollo en nuestro campo. El internacionalmente conocido Centro Proveedor de Documentos de la Biblio te ca Británica, es un ejem plode op ti mización de recursos bibliográficos, único en su género.

La in fraes truc tu ra para in ves ti ga ción en este cam po es bue na, generando gran cantidad de publicaciones científicas, tanto en bi blio te co lo gía como en in for ma ción. Mu chos de los tí tur los son clave para la profesión internacional, de los cuales da cuenta Library and Information Science Abstracts (LISA), un ín di ce in glés con si de ra do como nú me ro uno en su cla se.

Otra ventaja de las escuelas británicas es la cercanía que tienen con sus contrapartes de los demás países europeos, que juntos ofrecen un frente común para competir con el avance tecnológico de Estados Unidos; para la profesión esto es de enorme valía, porque cada nación europea ofrece diferentes sistemas informativos; sólo para mencionar los más avanzados, se deben nombrar a los países escandinavos, los alemanes y los fran ce ses que siem pre tie nen algo que ofre cer en los congresos de esta rama. 


\section{REFERENCIAS}

BOTTLE, R. T. “Education for IS or IT. Is there a difference?”. Journal of Information Science. (8)1984, 167-170.

BRAMLEY, G. Apprenticetograduate: a historyoflibraryeducation in the United King dom. Lon don: Cli ve Bin gley, $1981.218 \mathrm{p}$.

FANG, J. R. and P. Nau ta.InternationalGuidetoLibraryandInformationScience Education. Mun chen: K.G. Saur, 1985. $397-420$.

FENWICKE, W.H. "In for ma tion sys tems cu rricu lum. Edu ca tion for In for ma tion ma na ge ment: di rec tions for the fu tu re".Re cord of a Confe renceco-spon so red by the Information Institute, InternacionalAcademyat San ta Barba ra, and the Asso cia tion of Ame rican Library Schools, May 6-8, 1982. International Academy: Santa Barbara, 1983. 93-99.

GLEA VE, D.; C. An gell and K. Moo ley. "Struc tu ral chan ge within the in for mation pro fes sio n: a sce na rio for the 1990's".As lib Pro ceedings. 2(37)1985, 99-133.

THE LIBRARY ASSO CIA TION. Evidencesubmitted to the UGC/UAB transbinary group on librarianshipandinformationstudies. London: The Library Association, 1985. 25 p.

MCGARRY,K. "Progress in docu men ta tion.Edu ca tion for librarianship and in for mation scien ce: a re tros pect and re valua tion".Journal of Documentation, 39(2)1983, 95-122.

MO O RE, N. and E. Kempson. “The size and struc tu re of the li brary and in for ma tion workfor ce in the Uni ted King dom". Jour nal of Librarianship, 1(17)1985, 1-16.

MOORE, N. and E. Kempson. "The nature of the workforce". Journal of Librarianship 2(17)1985, $137-154$.

RENNIE, J. S. “A history of the Department of Information Science of The City University". Journal of Information Science, 12(12)1986, 3-13.

SAN TI NE LLI, P. "Chan ges on the books in the li brary schools". The times Higher Education Supplement" (pe rió di co). 25 Jul. 1986, p. 1.

SAUN DERS, W. L. "Pro fes sio nal edu ca tion, re search and de ve lopment". En: Saun ders, W. L.,Ed. BritishLibra rianship Today. London: The Library Association, 1976. 341-361.

THOMPSON, G. “The voice of the profession: the Library Association”. En Saunders, W. L. British Librarianship Today. London: The Library Association, 1976. 1-14

WIL SON, T.D. No tas de la con fe ren cia so bre edu ca ción bri tá ni ca, ofre ci da en la Re u nión de Per so nal Biblio te ca rio, ce le bra da en Valencia, España, mayo de 1985.7 p. 


\section{APENDICE}

\section{Directorio de escuelas}

Cada entrada incluye en orden alfabético el nombre de la institución y de la escuela, di rec ción y gra dos ofre ci dos, se pa ra dos con una dia go nal de sus res pec ti vas es pe cia li da des; año de fun da ción (Fang), nú me ro de maes tros y to tal de alum nos de li cen cia tu ra, maes tría y doc to ra do (The Ti mes). Cabe men cio nar que se con tó como alum no por cada dos de me dio tiempo. Al fi nal de este apén di ce se en cuentra el significado de las abreviaturas inglesas de los grados y especialidades.

\section{Brighton Polytechnic}

Department of Librarianship

Falmer

Brighton BN1 5AG

England

Tel. (0273) 606622

$\nabla$ BA/LIL, MPhil

1947 - 9 maestros

$120 \mathrm{Lic}$

City of Birmingham Polytechnic

Department of Librarianship

Franchise St.

Perry Barr

Birmingham B42 2SU

England

Tel. (021) 3566911

$\nabla$ BA/L,PDip/LIS

1950 - 10 maestros

35 Lic, 47 Mtría.

The City University

Dept. of Information Science

Northampton Square

London EXIV OHB

England

Tel. (01) 2534399

$\nabla$ MSc/ISM PDip/IS, MA/LAA

MSc/ISI, MPhil, PhD

1961 - 7 maestros

95 Mtria, 5 Dr.

Ealing Coll. of Higher Educ.

School of Lib. and Inf. Studies

St. Mary's Road

Ealing, London W5 5RF

England

Tel. (01) 5794111

$\nabla$ BA/LIS, PDip/LIS

1949 - 11 maestros

38 Lic, 50 Mtría.

Leeds Polytechnic

School of Librarianship

Leighton Hall

Beckett Park

Leeds LS6 3QS

England

Tel. 0532754061 $\nabla$ BA/L, BSc/IS, PDip/L, Cert

$\mathrm{MA} / \mathrm{L}$

1947 - 21 maestros

69 Lic, 60 Mtría, 2 Dr.

Liverpool Polytechnic

School of Lib. and Inf. Science

79 Tuithebarn St.

Liverpool L2 2ER

England

Tel. 00512271781

$\nabla$ BA/L, PDip/L, MPhil, PhD

1962 - ? maestros

119 Lic, 33 Mtría.

Loughborough Univ. of Tech.

Dept. of Lib. and Inf. Studies

Loughborough

Leicestershire LE11 3TU

England

Tel (0509) 263171

Telex 34319

$\nabla$ BA/LIS, BSc/LIS, PDip/LIS,

MA/LIS, MA/A, MA/ALISE, MSc/IS

MSc/LIS, MLS, MPhil, PhD

1972 - 21 maestros

106 Lic, 116 Mtría, ? Dr.

Loughborough Technical College

School of Librianship

Radmoor

Loughborough

Leicestershire LE11 3BT

England

Tel. (0509) 215831

$\nabla$ Dip (nivel Técnico)

1946 - 8 maestros

51 técnicos

Manchester Polytechnic

Dept. of Lib. and Inf. Stud.

Ormond Building

Lower Ormond St.

Manchester M15 6BX

England

Tel. (061) 2286171, Ext. 2696

$\nabla$ BA/LS, BA/L, PDip/L, MA/LS,

PCert

1947 - 16 maestros

198 Lic, 33 Mtría.

Newcastle Upon Tyne Polytechnic

School of Lib. and Inf. Stud.

Lipman Building

Ellison Place 
Newcastle upon Tyne NE1 8ST England

Tel. (0632) 612181, Ext. 15

$\nabla$ BA/L, PDip/LIS, MA/L 1947 - 17 maestros 146 Lic, 53 Mtria.

The Polytec. of North London School of Lib. and Inf. Studies 207-225 Essex Rd

London N1 3PN

England

Tel. (01) 6072789

Telex 25228

$\nabla$ BA/LIS, PDip/L, Fell, MPhil phD 1946 - 17 maestros 94 Lic, 112 Mtría, 6 Dr.

R. Gordon's Inst. of Technology School of Librianship

St. Andrew St.

Aberdeen

Scotland

United Kingdom

Tel. (0224) 633611

$\nabla$ BA/LIS, PDip/L, MPhil

1967 - 10 maestros

89 Lic, 27 Mtría.

The Queens Univ. of Belfast

Dept. of Lib. and Inf. Stud.

University Road

Belfast BT1 NN

Northern Ireland

United Kingdom

Tel. (232) 45133

Telex 74487 (Queens)

$\nabla$ BA/LIS, PDip/LIS, MA/IS, MLS

1963 - 8 maestros

40 Lic, 29 Mtría.

University College London

School of Lib. Archive, and

Inf. Studies

Gower St.

London WC 1E 6BT

England

Tel. (01) 3877050 $\nabla$ PDip/LIS, PA/LIS, PhD

1919 - 7 maestros

64 Mtría, 7 Dr.

University of Sheffield

Dept. of Information Studies

Western Bank

Sheffield S10 2TN

England

Tel. (0742) 78555, Ext.

Telex 547216 UGSHEF G

$\nabla$ MA/L, MA-ME/L, MSc/IS-Ciencias, MSc/IS-C.Soc, MPhil, PHD

1964 - 9 maestros

69 Mtría, 19 Dr.

University of Strathclyde

Dept. of Information Studies

Livingstone Tower

26 Richmond St.

Glasgow GL $1 \mathrm{XH}$

Scotland, United Kingdom

Tel. (041) 5524400

$\nabla$ BA/L, PDip/L, MA/LIS, MSc/IS,

$\mathrm{PhD}$

1945 - 16 maestros

73 Lic, 37 Mtría

University of Wales

College of Librarianship Wales

Llanbadarn Fawr

Aberystwyth

Dyfed SY23 3AS

Wales, United Kingdom

Tel. (0970) 3181

Telex 35391 (CLWG)

$\nabla$ BA/LIS, PDip/LIS, MA/LIS, Fell, PhD

1964 - 35 maestros

163 Lic, 161 Mtría, 4 Dr. 
Significado de abreviaturas

BA: Licenciatura en Bibliotecología

/L: en bibliotecología y en otra área

/LIS: en bib. y ciencias de la información

/LS: en ciencia bibliotecaria

BSc: Lic. en ciencias o ciencias sociales

Dip: Diploma en bibliotecología a nivel técnico

Fell: Supervisión para tomar el Fellowship de la LA

MA: Maestría en bibliotecología

/A: en archivos

/ALISE: archivos, bib. y ciencias de la inf.

/IS: en ciencias de la información

/L: en bibliotecología

/LIS: en bibliotecología y ciencias de la inf.
/LAA

/ME/L: Maestría para estudiantes con experiencia

MLS: Maestría en ciencia bibliotecaria, de año y medio MPhil: Maestría en filosofía

MSc: Maestría en ciencias o ciencias sociales

/IS: en ciencias de la información

/IST: en información de ciencia y tecnología

/LIS: en bib. y ciencias de la información

PCert: diploma en postgrado

/LIS: en bibliotecología y ciencias de la inf.

/IS: en ciencias de la información

PhD: Doctorado en Filosofía

? : Datos no proporcionados por la escuela. 